\title{
THE JENSEN COVERING PROPERTY
}

\author{
E. SCHIMMERLING AND W.H. WOODIN
}

\section{INTRODUCTION}

The Jensen covering lemma says that either $L$ has a club class of indiscernibles, or else, for every uncountable set $A$ of ordinals, there is a set $B \in L$ with $A \subseteq B$ and $\operatorname{card}(B)=\operatorname{card}(A)$. One might hope to extend Jensen's covering lemma to richer weasels, that is, to inner models of the form $L[\vec{E}]$ where $\vec{E}$ is a coherent sequence of extenders of the kind studied in Mitchell-Steel [MiSt]. The papers [MiSt], [St3], and SchSt1 show how to construct weasels with Woodin cardinals and more. But, as Prikry forcing shows, one cannot expect too direct a generalization of Jensen's covering lemma to weasels with measurable cardinals.

Recall from $[\mathrm{MiSt}$ that if $L[\vec{E}]$ is a weasel and $\alpha$ is an ordinal, then either $E_{\alpha}=\emptyset$, or else $E_{\alpha}$ is an extender over $J_{\alpha}^{\vec{E}}$. Let us say that $L[\vec{E}]$ is a lower-part weasel iff for every ordinal $\alpha, E_{\alpha}$ is not a total extender over $L[\vec{E}]$. In other words, if $L[\vec{E}]$ is a lower-part weasel, then no cardinal in $L[\vec{E}]$ is measurable as witnessed by an extender on $\vec{E}$. But a lower-part weasel $L[\vec{E}]$ could be rich in the sense that it may have levels $J_{\alpha}^{\vec{E}}$ satisfying ZFC + "there are many Woodin cardinal". We do not impose any bounds on the large cardinal axioms true in the levels of a lower-part weasel.

In this paper we show that if $L[\vec{E}]$ is an iterable, lower-part weasel, then either $L[\vec{E}]$ has indiscernibles, or else $L[\vec{E}]$ satisfies the Jensen covering property. The iterability and indiscernibility that we mean will be made precise in due course. Our result says, in a new way, that Prikry forcing is the essential limitation on extensions of Jensen's covering lemma.

Date: March 13, 1996.

1991 Mathematics Subject Classification. 03E.

Key words and phrases. large cardinals, core models, covering.

The research of the first author was partially supported by an NSF Mathematical Sciences Postdoctoral Research Fellowship at the Massachusetts Institute of Technology.

The research of the second author was partially supported by the NSF.. 
This paper is organized as follows. In $\S \S 2-5$, we prove several theorems regarding successor cardinals in lower-part weasels. Those in $\S 2$ and $\S 3$ might be seen as corollaries to the proofs of the main theorems in MiSchSt and MiSch respectively. Iterability is discussed in $\S 4$, where we sketch why internal strategic iterability for countable realizable premice suffices for all of the results in this paper. In $\S 5$, we prove our extension to Jensen's covering lemma.

That Theorem 2.4 is a corollary to [MiSchSt] was observed by Woodin $\mathbb{W W}$, who used it to define a core model based on the failure of $L(\mathbb{R})$ determinacy. We were originally and primarily motivated by this application and give a rough description of it at the end of $\S 2$.

The results presented in this paper build directly on the earlier work of Mitchell, Schimmerling, and Steel.

\section{Successor CARdinals With COUntable Closure}

We assume that the reader is familiar with [MiSt] and [St2], and adopt the notation established there. Throughout the paper, if $\pi$ is an embedding, then we shall write $\mathcal{E}(\pi)$, instead of the more common notation $E_{\pi}$, for the long extender derived from $\pi$. For the purposes of this paper, an iteration tree is a normal, padded iteration tree. If $\mathcal{T}$ is an iteration tree of successor length, then we shall write $\mathcal{M}_{\infty}(\mathcal{T})$ for the final model of $\mathcal{T}$. If $\mathcal{M}$ and $\mathcal{N}$ are premice, then $\mathcal{M} \unlhd \mathcal{N}$ means that $\mathcal{M}$ is an initial segment of $\mathcal{N}$, and $\mathcal{M} \triangleleft \mathcal{N}$ means that $\mathcal{M}$ is a proper initial segment of $\mathcal{N}$.

Definition 2.1. A premouse $\mathcal{M}$ is a lower-part premouse if and only if $\mathcal{M}$ is passive and $\dot{E}_{\alpha}^{\mathcal{M}}$ is not a total extender over $\mathcal{M}$ whenever $\alpha<\mathrm{OR}^{\mathcal{M}}$.

Definition 2.2. Let $\mathcal{M}$ be a premouse. Then $\mathcal{M}$ is iterable iff $\mathcal{M}$ is $\alpha$-iterable for every ordinal $\alpha$ in the sense of [St2, Definition 2.8]. That is, $\mathcal{M}$ is $\alpha$-iterable iff player II has a winning strategy in the iteration game $\mathcal{G}^{*}(\mathcal{M}, \alpha)$ of length $\alpha$ on $\mathcal{M}$. If $\alpha$ is an ordinal and $\kappa<\mathrm{OR}^{\mathcal{M}}$, then $\mathcal{M}$ is $\alpha$-iterable above $\kappa$ iff player II has a winning strategy in the iteration game of length $\alpha$ on $\mathcal{M}$ where player $I$ is restricted to playing extenders with critical points $\geq \kappa$. $\mathcal{M}$ is iterable above $\kappa$ iff $\mathcal{M}$ is $\alpha$-iterable above $\kappa$ for all ordinals $\alpha$.

Definition 2.3. $\mathcal{M}$ is an $x$-premouse iff $x$ is a set of ordinals and $\mathcal{M}$ is a premouse built over $x$.

Definition 2.3 means, among other things, that if $E$ is an extender from the $\mathcal{M}$-sequence, then $\cup x<\operatorname{crit}(E)$. All definitions involving premice have the obvious extensions to $x$-premice. 
Recall that a cardinal $\kappa$ is countably closed iff $\mu^{\aleph_{0}}<\kappa$ whenever $\mu<\kappa$.

Theorem 2.4. Suppose that $W=L[\vec{E}, x]$ is an iterable, lower-part $x$ weasel. Let $\kappa$ be a cardinal of $W$ such that $\cup x<\operatorname{card}(\kappa)$ and $\operatorname{card}(\kappa)$ is a countably closed cardinal. Put $\lambda=\left(\kappa^{+}\right)^{W}$ and suppose that

$$
\operatorname{cf}(\lambda)<\operatorname{card}(\kappa)
$$

Then, given $\delta<\operatorname{card}(\kappa)$, there exists $\mu \geq \delta$ and a normal, countably complete ultrafilter $U$ on $\mathcal{P}(\mu) \cap W$ such that $U$ is weakly amenable to $W$.

Definition 2.5. An iteration tree $\mathcal{T}$ is trivial iff $E_{\eta}^{\mathcal{T}}=\emptyset$ whenever $\eta<\operatorname{lh}(\mathcal{T})$. That is, $\mathcal{T}$ is trivial iff $\mathcal{T}$ consists exclusively of padding.

Definition 2.6. An iteration tree $\mathcal{T}$ is dropping iff $\eta+1 \in \mathcal{D}^{\mathcal{T}}$ whenever $0=\operatorname{pred}^{\mathcal{T}}(\eta+1)$.

Lemma 2.7. If $\mathcal{T}$ is an iteration tree on a lower part-part premouse and $E_{0}^{\mathcal{T}} \neq \emptyset$, then $\mathcal{T}$ is dropping.

Proof of Lemma 2.7. Since $\mathcal{M}_{0}^{\mathcal{T}}$ is a lower-part premouse, we have that $1 \in \mathcal{D}^{\mathcal{T}}$. Suppose that $\operatorname{pred}^{\mathcal{T}}(\eta+1)=0<\eta$ but $\eta+1 \notin \mathcal{D}^{\mathcal{T}}$. Then a simple calculation shows that

$$
\operatorname{crit}\left(E_{\eta}^{\mathcal{T}}\right)<\rho_{\omega}\left(\left(\mathcal{M}_{1}^{*}\right)^{\mathcal{T}}\right) \leq \operatorname{crit}\left(E_{0}^{\mathcal{T}}\right)<\operatorname{lh}\left(E_{0}^{\mathcal{T}}\right)<\operatorname{lh}\left(E_{\eta}^{\mathcal{T}}\right) .
$$

So $\operatorname{crit}\left(E_{0}^{\mathcal{T}}\right)$ is a limit of generators of $E_{\eta}^{\mathcal{T}}$. By the initial segment condition on good extender sequences, there is are initial segments of $E_{\eta}^{\mathcal{T}}\left\lceil\operatorname{crit}\left(E_{0}^{\mathcal{T}}\right)\right.$ on the $\mathcal{M}_{0}^{\mathcal{T}}$-sequence, and these initial segments are total extenders over $\mathcal{M}_{0}^{\mathcal{T}}$. But $\mathcal{M}_{0}^{\mathcal{T}}$ is a lower-part premouse, a contradiction.

Definition 2.8. An iteration tree $\mathcal{T}$ is thorough iff $E_{\eta}^{\mathcal{T}} \neq \emptyset$ and

$$
\mathcal{M}_{\eta}^{\mathcal{T}} \| \operatorname{lh}\left(E_{\eta}^{\mathcal{T}}\right)
$$

is a lower part premouse whenever $\eta<\operatorname{lh}(\mathcal{T})$.

Definition 2.9. An extender $E$ is a measure iff the only generator of $E$ is $\operatorname{crit}(E)$. 
Lemma 2.10. Suppose that $\mathcal{T}$ is a thorough iteration tree. Then $E_{\eta}^{\mathcal{T}}$ is a measure whenever $\eta<\operatorname{lh}(\mathcal{T})$, so $\mathcal{T}$ is linear. Moreover, $\mathcal{T}$ has the following very simple form:

$$
(\gamma+1, \eta]_{T} \cap \mathcal{D}^{\mathcal{T}}=\emptyset \Longrightarrow E_{\eta}^{\mathcal{T}}=i_{\gamma, \eta}^{\mathcal{T}}\left(E_{\gamma}^{\mathcal{T}}\right)
$$

whenever $\gamma<\eta<\operatorname{lh}(\mathcal{T})$.

Proof of Lemma 2.10. The first assertion is clear from the initial segment condition on $\mathcal{M}_{\eta}^{\mathcal{T}}$ and the thoroughness of $\mathcal{T}$. Suppose that $\gamma<\eta<\operatorname{lh}(\mathcal{T})$. If

$$
\operatorname{lh}\left(E_{\eta}^{\mathcal{T}}\right)>\operatorname{lh}\left(i_{\gamma, \eta}^{\mathcal{T}}\left(E_{\gamma}^{\mathcal{T}}\right)\right)
$$

then $i_{\gamma, \eta}^{\mathcal{T}}\left(E_{\gamma}^{\mathcal{T}}\right)$ witnesses that $\mathcal{M}_{\eta}^{\mathcal{T}} \| \operatorname{lh}\left(E_{\eta}^{\mathcal{T}}\right)$ is not a lower-part premouse. Suppose that

$$
\operatorname{lh}\left(E_{\eta}^{\mathcal{T}}\right)<\operatorname{lh}\left(i_{\gamma, \eta}^{\mathcal{T}}\left(E_{\gamma}^{\mathcal{T}}\right)\right)
$$

Then $E_{\eta}^{\mathcal{T}}$ witnesses that

$$
i_{\gamma, \eta}^{\mathcal{T}}\left(\mathcal{M}_{\gamma}^{\mathcal{T}} \| \operatorname{lh}\left(E_{\gamma}^{\mathcal{T}}\right)\right)
$$

is not a lower-part premouse. By elementarity, $\mathcal{M}_{\gamma}^{\mathcal{T}} \| \operatorname{lh}\left(E_{\gamma}^{\mathcal{T}}\right)$ is not a lower-part premouse, a contradiction.

Proof of Theorem 2.4. For the reader familiar with [MiSchSt, we remark that this proof begins just like the proof of the main result there. But the current proof is substantially simpler, as there is no need for an inductive proof that the lift-ups are strong.

Let $\Omega$ be a regular cardinal $>\lambda$, and let $X$ be an elementary substructure of $V_{\Omega}$ with

$$
\begin{gathered}
\operatorname{card}(X)<\operatorname{card}(\kappa), \\
{ }^{\omega} X \subset X, \\
\sup (X \cap \lambda)=\lambda,
\end{gathered}
$$

and

$$
(\cup x) \cup \delta \cup\left\{\mathcal{J}_{\lambda}^{W}\right\} \subset X
$$

Let $\pi: N \longrightarrow V_{\Omega}$ be the inverse of the transitive collapse of $X$. Say $\pi(\bar{\kappa})=\kappa, \pi(\bar{\lambda})=\lambda, \pi(\bar{W})=\mathcal{J}_{\lambda}^{W}$, and $\varepsilon=\operatorname{crit}(\pi)$. Then $\varepsilon<\kappa$.

If $W$ were 1-small, then we could quote [MiSt, Theorem 8.2] and conclude that $\bar{W}$ and $W$ agree below $\left(\varepsilon^{+}\right)^{\bar{W}}$. But $W$ is not assumed to satisfy a smallness condition. Nevertheless, we can argue as follows. Suppose that $\mathcal{M} \triangleleft \bar{W}$ with $\rho_{\omega}(\mathcal{M})=\varepsilon$. Let $(\overline{\mathcal{S}}, \mathcal{S})$ be a successful coiteration of $(\mathcal{M}, \pi(\mathcal{M}))$. All the models on $\overline{\mathcal{S}}$ and $\mathcal{S}$ agree below $\varepsilon$, and $\varepsilon$ is a cardinal in all the models on $\overline{\mathcal{S}}$ and $\mathcal{S}$ except possibly $\pi(\mathcal{M})$. Using the initial segment condition on good extender sequences and the fact that $\bar{W}$ is a lower-part $x$-premouse, one concludes that all 
extenders used on the iteration trees $\overline{\mathcal{S}}$ and $\mathcal{S}$ have critical points at least $\varepsilon$. Standard arguments using the soundness of $\mathcal{M}$ and $\pi(\mathcal{M})$ now show that both $\overline{\mathcal{S}}$ and $\mathcal{S}$ are trivial, so that $\mathcal{M} \triangleleft \pi(\mathcal{M})$. So every such $\mathcal{M}$ is a proper initial segment of $W$. Since the initial segments $\mathcal{M}$ of $\bar{W}$ with $\rho_{\omega}(\mathcal{M})=\varepsilon$ appear cofinally in $\bar{W} \|\left(\varepsilon^{+}\right)^{\bar{W}}$, we have shown that $\bar{W}$ and $W$ agree below $\left(\varepsilon^{+}\right)^{\bar{W}}$.

First, consider the case in which $\left(\varepsilon^{+}\right)^{\bar{W}}=\left(\varepsilon^{+}\right)^{W}$, so that $\bar{W}$ and $W$ have the same subsets of $\varepsilon$. Then Theorem 2.4 holds with $\mu=\varepsilon$ and $U$ equal to the measure derived from $\pi$, that is,

$$
U=\{x \in|\bar{W}| \mid x \subseteq \varepsilon \wedge \varepsilon \in \pi(x)\} .
$$

Thus, we may assume without loss of generality that $\left(\varepsilon^{+}\right)^{\bar{W}}<\left(\varepsilon^{+}\right)^{W}$.

Let $(\overline{\mathcal{T}}, \mathcal{T})$ be a successful coiteration of $(\bar{W}, W)$, and say this coiteration has length $\theta+1$. Note that both $\bar{W}$ and $W$ are both lower-part $x$-premice, and models of $\mathrm{ZF}^{-}$. Moreover, $\left(\varepsilon^{+}\right)^{\bar{W}} \leq \operatorname{lh}\left(E_{0}^{\mathcal{T}}\right)<\left(\varepsilon^{+}\right)^{W}$.

One of $\overline{\mathcal{T}}$ and $\mathcal{T}$ must be trivial, as otherwise

$$
[0, \theta]_{\bar{T}} \cap \mathcal{D}^{\overline{\mathcal{T}}} \neq \emptyset
$$

and

$$
[0, \theta]_{T} \cap \mathcal{D}^{\mathcal{T}} \neq \emptyset
$$

which leads to the usual contradiction as in the comparison lemma MiSt, Lemma 7.1]. Since $\bar{W}$ has fewer subsets of $\varepsilon$ than $W$, it must be that $\overline{\mathcal{T}}$ is trivial. Therefore $\bar{W} \triangleleft \mathcal{M}_{\theta}^{\mathcal{T}}$ and $\mathcal{T}$ does not involve any padding. By Lemma 2.7, since $W$ is a lower-part $x$-weasel, $\mathcal{T}$ is dropping. Since $\bar{W}$ is a lower-part $x$-premouse, $\mathcal{T}$ is thorough. Thus, $\mathcal{T}$ has the special form described in Lemma 2.10.

Let $\eta$ be the least ordinal such that $\nu_{\eta}^{\mathcal{T}}>\bar{\kappa}$, if such an ordinal exists, and let $\eta=\theta$ otherwise. We remark that because $\mathcal{T}$ is thorough and $\bar{\kappa}$ is a cardinal in $\bar{W}$,

$$
\nu_{\gamma}^{\mathcal{T}}>\bar{\kappa} \Longleftrightarrow \operatorname{crit}\left(E_{\gamma}^{\mathcal{T}}\right) \geq \bar{\kappa}
$$

for all $\gamma \leq \theta$.

Let $\mathcal{P}$ be the longest initial segment of $\mathcal{M}_{\eta}^{\mathcal{T}}$ with exactly those subsets of $\bar{\kappa}$ that are in $\bar{W}$. Then $\mathcal{P}$ is a $\bar{\kappa}$-sound $x$-premouse.

Let $\mathcal{R}=\operatorname{ult}(\mathcal{P}, \mathcal{E}(\pi)\lceil\kappa)$ and $\tilde{\pi}: \mathcal{P} \longrightarrow \mathcal{R}$ be the ultrapower map. Recall that this ultrapower is defined using coordinates $a \in[\kappa]^{<\omega}$ and certain $\mathcal{P}$-definable functions $f:[\kappa]^{<\omega} \longrightarrow|\mathcal{P}|$. The usual argument, using the countable completeness of $\mathcal{E}(\pi)\lceil\kappa$, shows that $\mathcal{R}$ is wellfounded. By the standard fine structural results in [MiSchSt, §2], $\mathcal{R}$ and $W$ agree below $\lambda$,

$$
\pi \uparrow \lambda=\tilde{\pi} \uparrow \lambda
$$




$$
\left(\kappa^{+}\right)^{\mathcal{R}}=\sup \left(\widetilde{\pi}^{\prime \prime} \bar{\lambda}\right)=\sup \left(\pi^{\prime \prime} \bar{\lambda}\right)=\pi(\bar{\lambda})=\lambda,
$$

and $\mathcal{R}$ is $\kappa$-sound.

We claim that if $\mathcal{P}$ is active, then $\dot{\mu}^{\mathcal{P}} \geq \bar{\kappa}$. For, if $\dot{\mu}^{\mathcal{P}}<\bar{\kappa}$, then initial segments of $\dot{F}^{\mathcal{P}}\lceil\bar{\kappa}$ are on the $\bar{W}$-sequence, contradicting that $\bar{W}$ is a lower-part $x$-premouse.

The previous claim implies that $\tilde{\pi}$ is continuous at $\left(\dot{\mu}^{+}\right)^{\mathcal{P}}$, and hence that $\dot{F}^{\mathcal{R}}$ measures all sets in $\mathcal{R}$. It follows that $\mathcal{R}$ is an $x$-premouse (rather than just an $x$-protomouse, in the terminology of MiSchSt]).

Countable completeness can also be used, in the standard way, to show that $\mathcal{R}$ is iterable above $\kappa$. Note that any coiteration of $(W, \mathcal{R})$ uses extenders with critical points $\geq \kappa$. Thus, we there is a successful coiteration of $(W, \mathcal{R})$, call it $(\overline{\mathcal{I}}, \mathcal{I})$.

It follows from the fact that $\mathcal{R}$ is $\kappa$-sound and $\left(\kappa^{+}\right)^{\mathcal{R}}=\left(\kappa^{+}\right)^{W}$, that $\overline{\mathcal{I}}$ is a trivial iteration. Thus, the coiteration has length $\mathrm{OR}$, and $W$ the lined up model of $\mathcal{I}$, by which we mean that

$$
\dot{E}^{W}=\bigcup\left\{\dot{E}^{\mathcal{M}_{\eta}^{\mathcal{S}}}\left\lceil\operatorname{lh}\left(E_{\eta}^{\mathcal{S}}\right) \mid \alpha \in \mathrm{OR}\right\}\right.
$$

Because $W$ is a lower-part $x$-weasel, it follows that $\mathcal{I}$ is thorough. In particular, $\mathcal{I}$ has the simple form described in Lemma 2.10.

Let $\alpha$ be an ordinal such that the drops of $\mathcal{I}$ are bounded in $\alpha$. Let $\mathcal{I}^{\prime}$ be the tail of $\mathcal{I}$ beginning from stage $\alpha$, and let $\mathcal{R}^{\prime}$ be the starting model of $\mathcal{I}^{\prime}$. Thus, $\mathcal{I}^{\prime}$ is a thorough iteration of

$$
\mathcal{R}^{\prime}=\mathcal{M}_{0}^{\mathcal{I}^{\prime}}=\mathcal{M}_{\alpha}^{\mathcal{I}}
$$

and $\mathcal{I}$ has no drops. Let

$$
C=\left\{\operatorname{crit}\left(E_{\beta}^{\mathcal{I}^{\prime}}\right) \mid \beta \in \mathrm{OR}\right\} .
$$

Then $C$ is a club class of indiscernibles for $W$. All sufficiently large cardinals are limit points of $C$. Let $\mu \in \lim (C)$ be a regular cardinal and

$$
U=\{A \in W \mid A \subseteq \mu \wedge \sup (A \cap C)=\mu\} .
$$

Then $\mu>\kappa>\delta$ and $U$ is a $\mu$-complete ultrafilter over $W$. Note that $U$ is just the measure whose trivial completion is the extender $E_{\mu}^{\mathcal{I}^{\prime}}$, so that $U$ is normal and weakly amenable to $W$.

We now briefly discuss how Theorem 2.4 comes up in the core model induction of Woodin [W]. For each rank $V_{\alpha}$ there is a certain lowerpart $x_{\alpha}$-weasel $W_{\alpha}$ such that $V_{\alpha} \subset W_{\alpha}$. Without giving the definition of $W_{\alpha}$, let us say that $W_{\alpha}$ is the closure of $V_{\alpha}$ under a certain inner model theory operator corresponding to the least level of $L(\mathbb{R})$ for which determinacy fails. As a result, $W_{\alpha} \cap H C$ is $L(\mathbb{R})$-definable and $W_{\alpha}$ is 
forcing absolute. I If there is an $\alpha$ such that $W_{\alpha}$ computes successors of countably closed, singular cardinals correctly, then $W_{\alpha}$ can reasonably be construed as the "true" core model. Suppose, on the other hand, that there is no such $\alpha$. Then, Theorem 2.4 implies that for every $\alpha$, there exists $\mu_{\alpha}>\alpha$ and a countably complete ultrafilter $U_{\alpha}$ on $\mathcal{P}\left(\mu_{\alpha}\right) \cap W_{\alpha}$ such that $U_{\alpha}$ is weakly amenable to $W_{\alpha}$. These properties of $U_{\alpha}$ are sufficient to develop the Steel core model theory [St2] in $W_{\alpha}$ up to $\mu_{\alpha}$. That is, we have a reasonable interpretation of $\left(K \| \mu_{\alpha}\right)^{W_{\alpha}}$. The failure of determinacy and the closure of $W_{\alpha}$ under lower parts implies that $\left(K \| \mu_{\alpha}\right)^{W_{\alpha}}$ does not reach a Woodin cardinal. In this case, it is

$$
\bigcup_{\alpha \in O R}(K \| \alpha)^{W_{\alpha}}
$$

that is taken as the "true" core model. Because $V_{\alpha} \subset W_{\alpha}$ for all $\alpha$, we can use [MiSchSt] to see that this "true" core model computes successors of countably closed, singular cardinals correctly.

Woodin [W] draws on [St1], [MaSt], [KW], [St2, MiSchSt, and Woodin's theorems on the close relationship between inner models with Woodin cardinals and the determinacy of games in $L(\mathbb{R})$.

It is possible to use Theorem 3.2 (below) and MiSch instead of Theorem 2.4 and MiSchSt, in the core model induction discussed above. This gives a core model that computes all successors of singular cardinals correctly, based on a failure of $L(\mathbb{R})$-determinacy.

\section{Successor Cardinals Without countable Closure}

In this section, we eliminate the hypothesis that $\operatorname{card}(\kappa)$ is countably closed from Theorem 2.4, and draw a somewhat weaker conclusion. We shall need a slightly stronger form of iterability.

Definition 3.1. An x-premouse $\mathcal{M}$ is $\star$-iterable iff $\mathcal{M}$ satisfies the following two conditions.

(a) For every ordinal $\alpha, \mathcal{M}$ is $(\omega, \alpha)$-iterable in the sense of [St2, Definition 2.8]. That is, for every $\alpha$, player II has a winning strategy in the almost normal iteration game $\mathcal{G}^{*}(\mathcal{M},(\omega, \alpha))$.

(b) There is no infinite composition of thorough iteration trees on $\mathcal{M}$ such that each iteration tree involves a drop.

The game $\mathcal{G}^{*}(\mathcal{M},(\omega, \alpha))$ mentioned in 3.1(a) is the variation of the usual iteration game on $\mathcal{M}$ which allows finitely many violations of the normality condition on iteration trees. Another way to express 3.1(b)

\footnotetext{
${ }^{1}$ Some examples of such weasel are given after the statement of Theorem 5.3.
} 
is to say that there is no infinite sequence $\mathcal{S}_{0} \frown \mathcal{S}_{1} \frown \ldots$ of thorough iteration trees such that $\mathcal{M}_{0}^{\mathcal{S}_{0}}=\mathcal{M}$, and $\mathcal{S}_{n}$ has successor length and

$$
\mathcal{M}_{0}^{\mathcal{S}_{n+1}} \triangleleft \mathcal{M}_{\infty}\left(\mathcal{S}_{n}\right)
$$

for all $n<\omega$. We remind the reader that thorough iteration trees are linear, and more, as was established in Lemma 2.1.

Theorem 3.2. Suppose that $W=L[\vec{E}, x]$ is an $\star$-iterable, lower-part $x$-weasel. Let $\kappa$ be a cardinal of $W$ such that

$$
(\cup x)^{+} \cup \aleph_{2} \leq \operatorname{card}(\kappa)
$$

Put $\lambda=\left(\kappa^{+}\right)^{W}$ and suppose that $\operatorname{cf}(\lambda)<\operatorname{card}(\kappa)$. Then, given

$$
\delta<\operatorname{card}(\kappa),
$$

there exists $\mu \geq \delta$ and a normal ultrafilter $U$ on $\mathcal{P}(\mu) \cap W$ such that $U$ is weakly amenable to $W$ and $\operatorname{ult}(W, U)$ is wellfounded. \&

Before giving the proof of Theorem 3.2, we isolate a useful property from the proof of Theorem 2.4.

Definition 3.3. $\Phi(\mathcal{M}, \mathcal{N})$ holds iff $\mathcal{M}$ is a lower-part $x$-premouse, $\mathcal{N}$ is an $x$-premouse, and there is a successful coiteration $(\overline{\mathcal{I}}, \mathcal{I})$ of $(\mathcal{M}, \mathcal{N})$ such that $\overline{\mathcal{I}}$ is trivial and $\mathcal{I}$ is thorough.

Notice that $\Phi(\mathcal{M}, \mathcal{N})$ holds iff $\mathcal{M}$ is a lower-part $x$-premouse, $\mathcal{N}$ is an $x$-premouse, and for every coiteration $(\overline{\mathcal{I}}, \mathcal{I})$ of $(\mathcal{M}, \mathcal{N})$, if $\overline{\mathcal{I}}$ is trivial and $\mathcal{I}$ is thorough, then

$$
\lim \left\langle\mathcal{M}_{\beta}^{\mathcal{I}} \mid \beta<\operatorname{lh}(\mathcal{I})\right\rangle
$$

is illfounded. Thus $\Phi(\mathcal{M}, \mathcal{N})$ is highly absolute.

Proof of Theorem 3.2. To do without countable closure, we shall use the same method as MiSch.

Let $\Omega>\lambda$ be a regular cardinal and

$$
\varepsilon=((\cup x) \cup \operatorname{cf}(\lambda) \cup \delta)^{+} .
$$

Then $\aleph_{2} \leq \varepsilon \leq \operatorname{card}(\kappa)$. Let $\left\langle X_{i} \mid i<\varepsilon\right\rangle$ be a continuous chain of elementary substructures of $V_{\Omega+1}$ such that for all $j<\varepsilon$,

$$
\begin{gathered}
\left(\operatorname{card}\left(X_{j}\right)\right)^{+}=\varepsilon, \\
\left\langle X_{i} \mid i \leq j\right\rangle \in X_{j+1},
\end{gathered}
$$

\footnotetext{
${ }^{2}$ From the proof of Theorem 3.2, one sees that $U$ can be chosen so that either $U$ is countably complete, or $U$ is the measure derived from an inverse transitive collapse $\pi: \bar{W} \longrightarrow W$. In the later case, ult $(\operatorname{ult}(\bar{W}, U), U)$ is wellfounded, since it embeds into ult $(W, U)$. This is useful in developing the Steel core model theory St2 within $\bar{W}$.
} 
and

$$
X_{j} \cap \varepsilon \in \varepsilon .
$$

We also have or assume that

$$
(\cup x) \cup \operatorname{cf}(\lambda) \cup \delta \cup\left\{\lambda, \mathcal{J}_{\Omega}^{W}\right\} \subset X_{0} .
$$

For $i<\varepsilon$, let $\varepsilon_{i}=X_{i} \cap \varepsilon_{i}$. Note that $\left\langle\varepsilon_{i} \mid i<\varepsilon\right\rangle$ is a normal sequence converging to $\varepsilon$. For $i<\varepsilon$, let $\pi_{i}: N_{i} \longrightarrow V_{\Omega+1}$ be the uncollapse of $X_{i}$. So $\operatorname{crit}\left(\pi_{i}\right)=\varepsilon_{i}$. Say $\pi_{i}\left(\kappa_{i}\right)=\kappa, \pi\left(\lambda_{i}\right)=\lambda$, and $\pi_{i}\left(W_{i}\right)=\mathcal{J}_{\Omega}^{W}$ whenever $i<\varepsilon$.

Case 1. There is a stationary set

$$
S_{0} \subseteq\left\{i<\varepsilon \mid \operatorname{cf}(i)>\omega \wedge i=\varepsilon_{i}\right\}
$$

such that $i \in S_{0}$ implies $\left(\varepsilon_{i}^{+}\right)^{W_{i}}<\left(\varepsilon_{i}^{+}\right)^{W}$.

In this case, we shall find a arbitrarily large ordinals $\mu$ and corresponding ultrafilters $U$ as in the conclusion of the theorem. Moreover, the ultrafilters will be countably complete.

For $i \in S_{0}$, let $\left(\overline{\mathcal{T}}_{i}, \mathcal{T}_{i}\right)$ be the successful coiteration of $\left(W_{i}, \mathcal{J}_{\Omega}^{W}\right)$, and say that this coiteration has length $\theta_{i}$. As in the proof of Theorem 2.4, $\overline{\mathcal{T}}_{i}$ is trivial, while $\mathcal{T}_{i}$ is dropping and thorough, whenever $i \in S_{0}$. In particular, $\Phi\left(W_{i}, \mathcal{J}_{\Omega}^{W}\right)$ holds for all $i \in S_{0}$.

For $i \in S_{0}$, let $\eta_{i}$ be the least ordinal $\eta$ such that $\nu_{\eta}^{\mathcal{T}_{i}}>\kappa_{i}$, if such an ordinal exists, and let $\eta_{i}$ be $\theta_{i}$ otherwise. And, also, let $\mathcal{P}_{i}$ be the

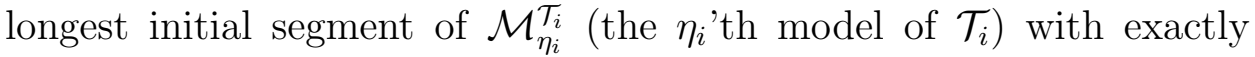
those subsets of $\kappa_{i}$ that are in $W_{i}$. Then, $\mathcal{P}_{i}$ is a $\kappa_{i}$-sound $x$-premouse whenever $i \in S_{0}$.

For $i \in S_{0}$, let $\mathcal{R}_{i}=\operatorname{ult}\left(\mathcal{P}_{i}, \mathcal{E}\left(\pi_{i}\right)\lceil\kappa)\right.$. At the corresponding point in the proof of Theorem 2.4, we used countable completeness to see that the corresponding ultrapower was wellfounded and iterable. But here, countable completeness is not available. Consider an arbitrary $i \in S_{0}$. If $\mathcal{R}_{i}$ is wellfounded, then it is a $\kappa$-sound $x$-premouse that agrees with $W$ below $\lambda$. If, in addition, $\mathcal{R}_{i}$ is iterable above $\kappa$, then $\Phi\left(W, \mathcal{R}_{i}\right)$ holds, giving us the indiscernibles for $W$, and we are done as in Theorem 2.4. Thus, we may assume that $\Phi\left(W, \mathcal{R}_{i}\right)$ fails for all $i \in S_{0}$.

Let $S$ be a stationary subset of $S_{0}$ on which the choice functions $i \mapsto \eta_{i}$ and $i \mapsto n\left(\mathcal{P}_{i}, \kappa_{i}\right)$ are constant. Such a set $S$ exists by Fodor's lemma MiSch, Lemma 1.1]. Say $\eta_{i}=\eta$ and $n\left(\mathcal{P}_{i}, \kappa_{i}\right)=n$ whenever $i \in S$.

So if $i \in S$ and $\mathcal{R}_{i}$ is wellfounded, then $n\left(\mathcal{R}_{i}, \kappa\right)=n, \mathcal{R}_{i}$ agrees with $W$ below $\lambda$, but $\mathcal{R}_{i}$ is not iterable above $\kappa$. Even if $\mathcal{R}_{i}$ is illfounded, $\lambda$ is in the wellfounded part of $\mathcal{R}_{i}$ and $\mathcal{R}_{i}$ agrees with $W$ below $\lambda$. (We 
remark that, without loss of generality, either $\mathcal{R}_{i}$ is not wellfounded for all $i \in S$, or $\mathcal{R}_{i}$ is wellfounded but not iterable for all $i \in S$.)

For the rest of the proof of the theorem under Case 1, fix

$$
j \in \lim (S) \cap S .
$$

Let $\psi: M \longrightarrow V_{\Omega+2}$ be elementary with $M$ countable and transitive, and everything relevant in the range of $\psi$. Say $\psi\left(x^{\prime}\right)=x, \psi\left(\Omega^{\prime}\right)=$ $\Omega, \psi\left(W^{\prime}\right)=\mathcal{J}_{\Omega}^{W}, \psi\left(\mathcal{P}^{\prime}\right)=\mathcal{P}_{j}, \psi\left(\mathcal{R}^{\prime}\right)=\mathcal{R}_{j}$, and $\psi\left(\kappa^{\prime}\right)=\kappa$. Then $\Phi\left(W^{\prime}, \mathcal{R}^{\prime}\right)$ fails in $M$, hence in $V$, so $\mathcal{R}^{\prime}$ is not iterable above $\kappa^{\prime}$ (possibly because $\mathcal{R}^{\prime}$ is not wellfounded).

Claim 3.2.1. $\operatorname{ran}(\psi) \cap \lambda$ is unbounded in $\lambda$.

Sketch of Claim 3.2.1. Suppose to the contrary that $\operatorname{ran}(\psi) \cap \lambda$ is bounded in $\lambda$. Let $\tilde{\pi}: \mathcal{P}_{j} \longrightarrow \mathcal{R}_{j}$ be the ultrapower map. Then $\tilde{\pi}^{\prime \prime} \lambda_{j}$ is unbounded in $\lambda$ and $\tilde{\pi} \in \operatorname{ran}(\psi)$. Moreover, the following diagram commutes.

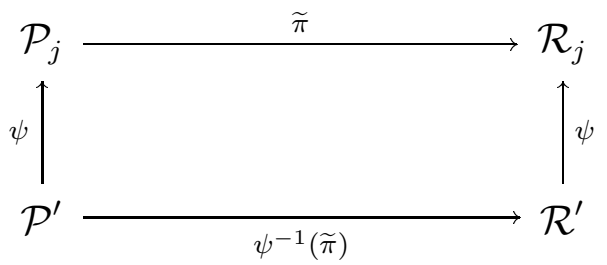

Therefore $\operatorname{ran}(\psi) \cap \lambda_{j}$ is bounded in $\lambda_{j}$.

We shall use [Sch1, §2] to find a proper initial segment $\mathcal{L}$ of $\mathcal{P}_{j}$ and an almost $\Sigma_{n+1}$-embedding $\varphi: \mathcal{L} \longrightarrow \mathcal{P}_{j}$ such that

$$
\operatorname{ran}(\psi) \cap\left|\mathcal{P}_{j}\right| \subset \operatorname{ran}(\varphi)
$$

$\varphi \in\left|\mathcal{P}_{j}\right|$, and

$$
\operatorname{ran}(\psi) \cap\left|\mathcal{R}_{j}\right| \subset \operatorname{ran}(\widetilde{\pi}(\varphi)) .
$$

Moreover, we shall have $\rho_{n+1}(\mathcal{L})=\kappa_{j}$ so that $\mathcal{L} \triangleleft \mathcal{P}_{j} \| \lambda_{j}$ and

$$
\tilde{\pi}(\mathcal{L}) \triangleleft \mathcal{R}_{j} \| \lambda .
$$

Then $\pi_{j}(\mathcal{L})=\tilde{\pi}(\mathcal{L})$ by the agreement of $\pi_{j}$ and $\tilde{\pi}$. So $\pi_{j}(\mathcal{L}) \triangleleft W$ by the agreement of $\mathcal{R}_{j}$ and $W$, Therefore $\pi_{j}(\mathcal{L})$ is iterable. 
We shall have the following diagram.

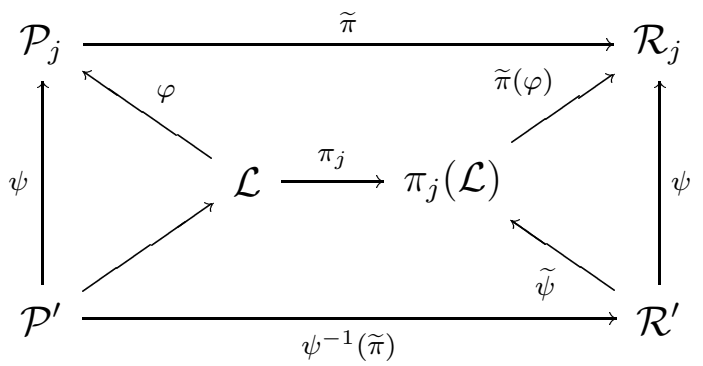

We have labeled $(\tilde{\pi}(\varphi))^{-1} \circ \psi$ as $\widetilde{\psi}$ in the diagram above. The map $\widetilde{\psi}$ is a weak $n$-embedding which is $\Sigma_{n+1}$-elementary from $\mathcal{R}^{\prime}$ into $\pi_{j}(\mathcal{L})$. Because $\pi_{j}(\mathcal{L})$ is iterable, the existence of a map such as $\psi$ implies that $\mathcal{R}^{\prime}$ is iterable. But $\mathcal{R}^{\prime}$ is not iterable above $\kappa^{\prime}$, while $\pi_{j}(\mathcal{L})$ is iterable. This is the desired contradiction.

It remains to construct $\varphi: \mathcal{L} \longrightarrow \mathcal{P}_{j}$ as described above. Let $\rho=$ $\rho_{n}\left(\mathcal{P}_{j}\right)$. For notational simplicity alone, let us restrict our attention to the case $n=0$ and $\mathcal{P}_{j}$ is not type III, so that $\rho=\mathrm{OR}^{\mathcal{P}_{j}}$ (the general case can be handled similarly by working over the $\Sigma_{n}$ coding structure of $\mathcal{P}_{j}$, as is usual in Jensen fine structure). In the proof of Sch1, Theorem 2.9], it is shown how to obtain a parameter $r$ in $\mathcal{P}_{j}$ such that $p_{1}\left(\mathcal{P}_{j}\right)$ is included in $r$, with the property that for all $\alpha<\kappa_{j}$, if we set

$$
\bar{\rho}(\alpha)=\sup \left(H_{1}^{\mathcal{P}_{j}}(\alpha \cup r) \cap \rho\right)
$$

and

$$
\bar{\lambda}(\alpha)=\sup \left(H_{1}^{\mathcal{P}_{j}}(\alpha \cup r) \cap \lambda_{j}\right)
$$

then

$$
\bar{\rho}(\alpha)<\rho \Longleftrightarrow \bar{\lambda}(\alpha)<\lambda_{j} .
$$

Without repeating the details, let us remark that the main facts used in defining $r$ are the uncountable cofinality of $\lambda_{j}$ and the $\kappa_{j}$-soundness of $\mathcal{P}_{j}$.

For any $\alpha<\kappa_{j}$, let

$$
\mathcal{L}_{\alpha}=\mathcal{H}_{1}^{\mathcal{P}_{j} \mid \bar{\rho}(\alpha)}(\bar{\lambda}(\alpha) \cup r)
$$

and

$$
\varphi_{\alpha}: \mathcal{L}_{\alpha} \longrightarrow \mathcal{P}_{j}
$$

be the inverse transitive collapse. 
In what follows, we consider mainly $\alpha<\kappa_{j}$ for which $\mathcal{L}_{\alpha} \neq \mathcal{P}_{j}$. Equivalently, $\bar{\rho}(\alpha) \neq \rho$. Equivalently, $\bar{\lambda}(\alpha) \neq \lambda_{j}$. Call such $\alpha$ reasonable. If $\alpha$ is reasonable, then $\varphi_{\alpha} \in\left|\mathcal{P}_{j}\right|$.

We claim that for all sufficiently large reasonable $\alpha<\kappa_{j}$, setting $\varphi=\varphi_{\alpha}$ and $\mathcal{L}=\mathcal{L}_{\alpha}$ works.

We first argue that for all $\alpha<\kappa_{j}, \mathcal{L}_{\alpha}$ is an $x$-premouse. In the notation of [Sch1], $\varphi_{\alpha}$ is an almost $\Sigma_{1}$-embedding. General facts about such embeddings reduce the problem of seeing that $\mathcal{L}_{\alpha}$ is an $x$-premouse to seeing that, if $\mathcal{P}_{j}$ is active, then $\dot{F}^{\mathcal{L}}{ }^{\alpha}$ measures all sets in $\mathcal{L}_{\alpha}$. In the terminology of MiSchSt, we must see that $\mathcal{L}_{\alpha}$ is an $x$-premouse, and not merely an $x$-protomouse. Note that this is a real concern, given that if $\mathcal{P}_{j}$ is active and $\sigma<\mathrm{OR}^{\mathcal{P}_{j}}$, then $\mathcal{P}_{j}\lceil\sigma$ is certainly not an $x$ premouse.

Suppose that $\mathcal{P}_{j}$ is active. In order to see that $\dot{F}^{\mathcal{L}_{\alpha}}$ measures all sets in $\mathcal{L}_{\alpha}$, it is enough to see that $\dot{\mu}^{\mathcal{P}_{j}} \geq \kappa_{j}$. Suppose to the contrary that $\dot{\mu}^{\mathcal{P}_{j}}<\kappa_{j}$. Since $\lambda_{j}$ is a cardinal in $\mathcal{P}_{j}$, by the initial segment condition on $\mathcal{P}_{j}$, there is a proper initial segment $G$ of $\dot{F}^{\mathcal{P}_{j}}$ which is on the $\mathcal{P}_{j}$ sequence below $\lambda_{j}$. Recall that $\mathcal{P}_{j}$ agrees with $W_{j}$ below $\lambda_{j}$ and that $\lambda_{j}$ is the successor cardinal of $\kappa_{j}$ in both $\mathcal{P}_{j}$ and $W_{j}$. Therefore, $G$ is an extender on the $W_{j}$-sequence which measures all sets in $W_{j}$. This is a contradiction because $W_{j}$ is a lower-part $x$-weasel.

We next claim that $\mathcal{L}_{\alpha} \triangleleft \mathcal{P}_{j}$ for sufficiently large reasonable $\alpha<\kappa_{j}$. 9 First, since $r$ contains $p_{1}\left(\mathcal{P}_{j}\right)$ and $\mathcal{P}_{j}$ is $\kappa_{j}$-sound, for all sufficiently large reasonable $\alpha<\kappa$,

$$
\begin{gathered}
\operatorname{crit}\left(\varphi_{\alpha}\right)=\bar{\lambda}(\alpha), \\
\mathcal{L}_{\alpha} \text { is 1-sound, } \\
\rho_{1}\left(\mathcal{L}_{\alpha}\right)=\kappa_{j}, \\
\varphi_{\alpha}\left(p_{1}\left(\mathcal{L}_{\alpha}\right)\right)=p_{1}\left(\mathcal{P}_{j}\right),
\end{gathered}
$$

and

$$
p_{1}\left(\mathcal{L}_{\alpha}\right)=\left(\varphi_{\alpha}\right)^{-1}\left(p_{1}\left(\mathcal{P}_{j}\right)\right) .
$$

It enough to take $\alpha$ large enough so that $r$ and the solidity witnesses for $p_{1}\left(\mathcal{P}_{j}\right)$ are all elements of

$$
H_{1}^{\mathcal{P}_{j} \mid \bar{\rho}(\alpha)}\left(\kappa_{j} \cup p_{1}\left(\mathcal{P}_{j}\right)\right) .
$$

If $\mathcal{P}_{j}$ were 1-small, then we could apply the condensation result Sch1, Theorem 2.6] to see that $\mathcal{L}_{\alpha} \triangleleft \mathcal{P}_{j}$ for all sufficiently large reasonable $\alpha<\kappa_{j}$. But there is no smallness condition on $W$, hence not on $\mathcal{P}_{j}$, so a small argument is required.

\footnotetext{
${ }^{3}$ The analogous fact was overlooked in Sch1; a slightly simpler proof of Sch1, Theorem 2.9] is possible.
} 
The fact that $W_{j}$ is a lower-part $x$-weasel that agrees with $\mathcal{P}_{j}$ below $\lambda_{j}$ implies that in any coiteration of $\left(\mathcal{L}_{\alpha}, \mathcal{P}_{j}\right)$, no extender with critical point $<\kappa_{j}$ is used. Since $\mathcal{P}_{j}$ is iterable above $\kappa_{j}$, and the embedding $\varphi_{\alpha}: \mathcal{L}_{\alpha} \longrightarrow \mathcal{P}_{j}$ is sufficiently elementary, $\mathcal{L}_{\alpha}$ is iterable above $\kappa_{j}$. So there is a successful coiteration $(\overline{\mathcal{S}}, \mathcal{S})$ of $\left(\mathcal{L}_{\alpha}, \mathcal{P}_{j}\right)$. But now the $\kappa_{j}$ soundness of both $\mathcal{P}_{j}$ and $\mathcal{L}_{\alpha}$ can be used to see that $\mathcal{L}_{\alpha} \triangleleft \mathcal{P}_{j}$.

Now fix $\mathcal{L}=\mathcal{L}_{\alpha}$ and $\varphi=\varphi_{\alpha}$ for some reasonable $\alpha<\kappa_{j}$ which is large enough so that $\mathcal{L}_{\alpha} \triangleleft \mathcal{P}_{j}$ and

$$
\sup (\operatorname{ran}(\psi) \cap \rho)<\bar{\pi}(\alpha) .
$$

This last condition guarantees that $\operatorname{ran}(\psi) \cap\left|\mathcal{P}_{j}\right| \subset \operatorname{ran}(\varphi)$ and

$$
\operatorname{ran}(\psi) \cap\left|\mathcal{R}_{j}\right| \subset \operatorname{ran}(\widetilde{\pi}(\varphi)) .
$$

All the requirements of $\varphi$ and $\mathcal{L}$ set out at the beginning of the proof of Claim 3.2.1 are now plain, and so we are done with that claim.

We continue the proof of Theorem 3.2. Since $\psi$ is countable, there is an $i \in j \cap S$ such that $\operatorname{ran}(\psi) \cap X_{j} \subset X_{i}$. Here we are using that $j \in \lim (S)$ and $\operatorname{cf}(j)>\omega$. Fix such an $i$ for the rest of the proof of the theorem under Case 1. Let $\pi_{i, j}: N_{i} \longrightarrow N_{j}$ be the uncollapse of $\left(\pi_{j}\right)^{-1}\left(X_{i}\right)$. Since $\operatorname{ran}(\psi) \cap \lambda$ is unbounded in $\lambda$ (by Claim 3.2.1), we have that

$$
\sup \left(\left(\pi_{i, j}\right)^{\prime \prime} \lambda_{i}\right)=\pi_{i, j}\left(\lambda_{i}\right)=\lambda_{j} .
$$

Arguing as in MiSch, Lemma 2.1], we find an $x$-premouse $\mathcal{P}_{j}^{*}$ such that $\mathcal{P}_{j}^{*}$ agrees with $W_{i}$ below $\lambda_{i}$ and

$$
\mathcal{P}_{j}=\operatorname{ult}\left(\mathcal{P}_{j}^{*}, \mathcal{E}\left(\pi_{i, j}\right)\left\lceil\kappa_{j}\right)\right.
$$

Moreover, $\left(\left(\kappa_{i}\right)^{+}\right)^{\mathcal{P}_{j}^{*}}=\lambda_{i}$ and $\mathcal{P}_{j}^{*}$ is $\kappa_{i}$-sound with $n\left(\mathcal{P}_{j}^{*}, \kappa_{i}\right)=n$. In MiSch, $\mathcal{P}_{j}^{*}$ is what is called the "pull-back" of $\mathcal{P}_{j}$ by $\pi_{i, j}$.

We claim that $\mathcal{P}_{i}=\mathcal{P}_{j}^{*}$. Note that any coiteration of $\mathcal{P}_{i}$ and $\mathcal{P}_{j}^{*}$ involves only extenders with critical points at least $\kappa_{i}$. This follows from the initial segment condition, using the fact that both $\mathcal{P}_{i}$ and $\mathcal{P}_{j}^{*}$ are premice which agree with $W_{i}$ up to $\lambda_{i}, W_{i}$ is a lower-part $x$-premouse, and $\lambda_{i}$ is the cardinal successor of $\kappa_{i}$ in all three premice. Since both $\mathcal{P}_{i}$ and $\mathcal{P}_{j}^{*}$ are iterable above $\kappa_{i}$, there is a successful coiteration $(\overline{\mathcal{S}}, \mathcal{S})$ of $\left(\mathcal{P}_{i}, \mathcal{P}_{j}^{*}\right)$. But now the standard argument using the $\kappa_{i}$-soundness of $\mathcal{P}_{i}$ and $\mathcal{P}_{j}^{*}$ shows that the coiteration is trivial, and hence $\mathcal{P}_{i}=\mathcal{P}_{j}^{*}$.

Recall that $\mathcal{R}_{i}=\operatorname{ult}\left(W_{i}, \mathcal{E}\left(\pi_{j}\right)\lceil\kappa)\right.$ and that $\Phi\left(W, \mathcal{R}_{i}\right)$ fails. In fact, $\Phi\left(\mathcal{J}_{\Omega}^{W}, \mathcal{R}_{i}\right)$ fails. So

$$
N_{j} \models \neg \Phi\left(\left(\pi_{j}\right)^{-1}\left(\mathcal{J}_{\Omega}^{W}\right),\left(\pi_{j}\right)^{-1}\left(\operatorname{ult}\left(W_{i}, \mathcal{E}\left(\pi_{i}\right)\lceil\kappa)\right)\right) .\right.
$$

But $\pi_{j}\left(W_{j}\right)=\mathcal{J}_{\Omega}^{W}$,

$$
\pi_{j}\left(\pi_{i, j}\left\lceil\kappa_{j}\right)=\pi_{i}\lceil\kappa\right.
$$


and, as we have seen,

$$
\mathcal{P}_{j}=\operatorname{ult}\left(\mathcal{P}_{i}, \mathcal{E}\left(\pi_{i, j}\right)\left\lceil\kappa_{j}\right)\right.
$$

Therefore

$$
N_{j} \models \neg \Phi\left(W_{j}, \mathcal{P}_{j}\right) .
$$

By upward absoluteness, $\Phi\left(W_{j}, \mathcal{P}_{j}\right)$ fails.

But notice that $\Phi\left(W_{j}, \mathcal{P}_{j}\right)$ holds trivially, since $P_{j}$ is an initial segment of a model on $\mathcal{T}_{j}$ and $\left(\overline{\mathcal{T}}_{j}, \mathcal{T}_{j}\right)$ is a coiteration of $\left(W_{j}, W\right)$. Thus, we have a contradiction, and the proof of Theorem 3.2 under Case 1 is complete.

Case 2. There is a stationary set

$$
S \subseteq\left\{i<\varepsilon \mid \operatorname{cf}(i)>\omega \wedge i=\varepsilon_{i}\right\}
$$

such that $i \in S$ implies $\left(\varepsilon_{i}^{+}\right)^{W_{i}}=\left(\varepsilon_{i}^{+}\right)^{W}$.

In this case, we shall find an ordinal $\mu$ with $\delta<\mu<\operatorname{card}(\kappa)$, and an ultrafilter $U$ on $\mathcal{P}(\mu) \cap W$ such that $U$ is weakly amenable to $W$, and $\Omega$ contained in the wellfounded part of ult $(W, U)$. Since $\Omega$ can be taken arbitrarily large, and there are only set many possible ultrafilters $U$, there will be at least one $U$ that satisfies the conclusion of the theorem.

As in the proof of Theorem 2.4,W and $W_{i}$ have the same subsets of $\varepsilon_{i}$ whenever $i \in S$. Let $\mathcal{P}=\mathcal{J}_{\Omega}^{W}$. Note that $\mathcal{P}$ is a lower-part $x$-premouse, since $\Omega$ is a cardinal of $W$. Let $\mathcal{R}_{i}=\operatorname{ult}\left(W_{i}, \mathcal{E}\left(\pi_{i}\right)\lceil\varepsilon)\right.$ whenever $i \in S$. In other words, $\mathcal{R}_{i}$ is the ultrapower of $\mathcal{P}$ by the superstrong extender derived from $\pi_{i}$.

It is enough to see that there exists an $i \in S$ such that $\mathcal{R}_{i}$ is wellfounded, for the following reasons. Let $U_{i}$ be the measure derived from $\pi_{i}$, that is,

$$
U_{i}=\left\{x \in W_{i} \mid x \subseteq \varepsilon_{i} \wedge \varepsilon_{i} \in \pi_{i}(x)\right\} .
$$

Then $U_{i}$ is an ultrafilter over $\mathcal{P}\left(\varepsilon_{i}\right) \cap W$ and $U_{i}$ is weakly amenable to $W$. Also, there is an embedding of $\operatorname{ult}\left(\mathcal{P}, U_{i}\right)$ into $\mathcal{R}$. Therefore, if $\mathcal{R}_{i}$ is wellfounded, then so is $\operatorname{ult}\left(\mathcal{P}, U_{i}\right)$.

Assume towards a contradiction that $\mathcal{R}_{i}$ is illfounded whenever $i \in S$.

Fix $j \in S \cap \lim (S)$. We shall show that $\mathcal{R}_{j}$ is wellfounded by an argument similar to that used in Case 1, letting $\mathcal{P}$ play the role of $\mathcal{P}_{i}$ for all $i \in S$.

Let $\psi: M \longrightarrow V_{\Omega+2}$ be elementary with $M$ countable and transitive, and everything relevant in the range of $\psi$. Say $\psi\left(x^{\prime}\right)=x, \psi\left(\Omega^{\prime}\right)=\Omega$, $\psi\left(W^{\prime}\right)=\mathcal{J}_{\Omega}^{W}, \psi\left(\mathcal{P}^{\prime}\right)=\mathcal{P}, \psi\left(\mathcal{R}^{\prime}\right)=\mathcal{R}_{j}$, and $\psi\left(\kappa^{\prime}\right)=\kappa$. Then $\mathcal{R}^{\prime}$ is illfounded. 
For the rest of the proof of the theorem, fix $i \in j \cap S$ such that $\operatorname{ran}(\psi) \cap X_{j} \subset X_{i}$. Let $\pi_{i, j}: N_{i} \longrightarrow N_{j}$ be the uncollapse of $\left(\pi_{j}\right)^{-1}\left(X_{i}\right)$. Arguing as in [MiSch, Lemma 2.2], we find a "pull-back" $\mathcal{P}^{*}$ of $\mathcal{P}$ by $\pi_{i, j}$. That is, we find an $x$-premouse $\mathcal{P}^{*}$ such that $\mathcal{P}^{*}$ agrees with $W$ below

$$
\varepsilon^{*}=\left(\left(\varepsilon_{i}\right)^{+}\right)^{\mathcal{P}^{*}},
$$

and the following commutative diagram.

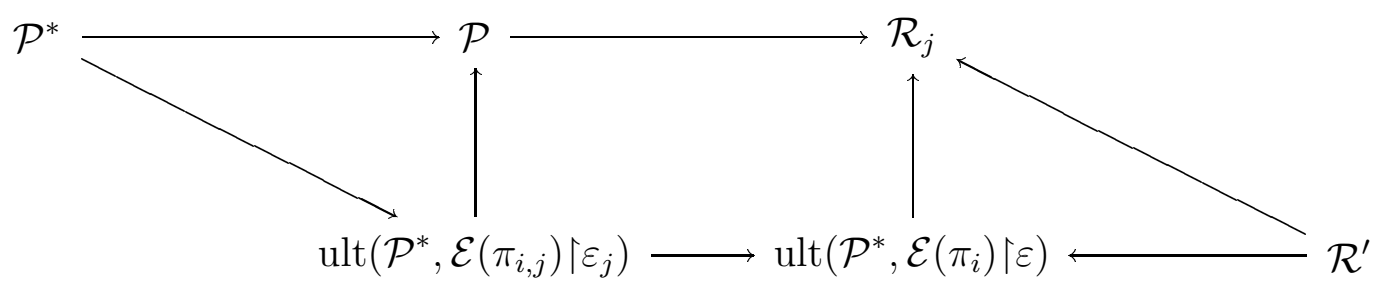

Thus ult $\left(\mathcal{P}^{*}, \mathcal{E}\left(\pi_{i, j}\right)\left\lceil\varepsilon_{j}\right)\right.$ is wellfounded and iterable, while ult $\left(\mathcal{P}^{*}, \mathcal{E}\left(\pi_{i}\right)\lceil\varepsilon)\right.$ is illfounded.

Recall that $\mathcal{R}_{i}$ is illfounded. Therefore

$$
N_{j} \models " \pi_{j}^{-1}\left(\mathcal{R}_{i}\right) \text { is illfounded". }
$$

Hence

$$
\pi_{j}^{-1}\left(\mathcal{R}_{i}\right)=\operatorname{ult}\left(\pi_{j}^{-1}(\mathcal{P}), \mathcal{E}\left(\pi_{i, j}\right)\left\lceil\varepsilon_{j}\right)\right.
$$

is illfounded. The shift lemma gives us the following diagram.

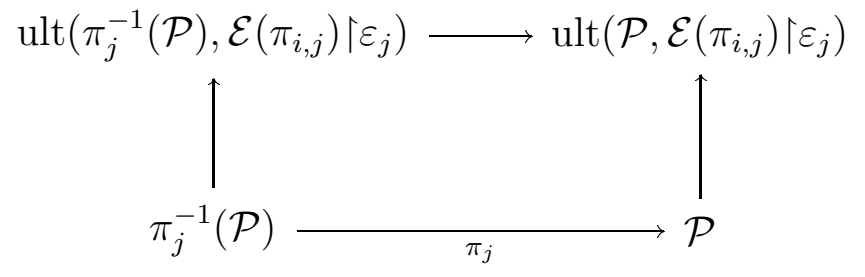

Thus both $\mathcal{R}_{i}=\operatorname{ult}\left(\mathcal{P}, \mathcal{E}\left(\pi_{i}\right)\lceil\varepsilon)\right.$ and $\operatorname{ult}\left(\mathcal{P}, \mathcal{E}\left(\pi_{i, j}\right)\left\lceil\varepsilon_{j}\right)\right.$ are illfounded.

We define a "minimal" $x$-premouse $\mathcal{Q}$ as follows. This is where $\star-$ iterability is used rather than just iterability. Let $\mathcal{Q}_{0}$ be the least initial segment $\mathcal{Q}^{\prime}$ of $\mathcal{P}$ above $\varepsilon_{i}$ such that $\mathcal{Q}^{\prime}$ is a lower-part $x$-premouse and $\operatorname{ult}\left(\mathcal{Q}^{\prime}, \mathcal{E}\left(\pi_{i}\right)\lceil\varepsilon)\right.$ is illfounded. Suppose that $0<\ell<\omega$ and for all $k<\ell$, we have defined $\mathcal{Q}_{k+1}$ and a non-trivial, thorough iteration tree $\mathcal{S}_{k+1}$ 
on $\mathcal{Q}_{k}$ such that $\mathcal{Q}_{k+1} \unlhd \mathcal{M}_{\infty}\left(\mathcal{S}_{k+1}\right)$. If there is a non-trivial thorough iteration tree $\mathcal{S}$ of successor length on $\mathcal{Q}_{\ell}$ such that all critical points of extenders used on $\mathcal{S}$ are at least $\varepsilon_{i}$, and there is a proper initial segment $\mathcal{Q}^{\prime}$ of $\mathcal{M}_{\infty}(\mathcal{S})$ such that $\mathcal{Q}^{\prime}$ is a lower-part $x$-premouse and $\operatorname{ult}\left(\mathcal{Q}^{\prime}, \mathcal{E}\left(\pi_{i}\right)\lceil\varepsilon)\right.$ is illfounded, then let $\mathcal{S}_{\ell+1}$ and $\mathcal{Q}_{\ell+1}$ be the least such $\mathcal{S}$ and $\mathcal{Q}^{\prime}$. I Otherwise, leave $\mathcal{S}_{\ell+1}$ and $\mathcal{Q}_{\ell+1}$ undefined. By the $\star-$ iterability of $W$ (because there is no infinite composition of thorough iteration trees on $W$ such that each iteration tree involves a drop), there is a $k<\omega$ such that $\mathcal{Q}_{k+1}$ is not defined. Let $\mathcal{Q}=\mathcal{Q}_{k}$.

We have that $\mathcal{Q}$ is a lower-part $x$-premouse, and $\mathcal{Q}$ is iterable since $W$ is $\star$-iterable. Note that $\operatorname{ult}\left(\mathcal{Q}, \mathcal{E}\left(\pi_{i}\right)\lceil\varepsilon)\right.$ is illfounded, while if $\mathcal{S}$ is any thorough iteration tree on $\mathcal{Q}$ with critical points at least $\varepsilon_{i}$ and $\mathcal{Q}^{\prime}$ is any proper initial segment of $\mathcal{M}_{\infty}(\mathcal{S})$, then $\operatorname{ult}\left(\mathcal{Q}^{\prime}, \mathcal{E}\left(\pi_{i}\right)\lceil\varepsilon)\right.$ is wellfounded. Also note that $\mathcal{Q} \in X_{j}$. Essentially the same argument that showed that

$$
\operatorname{ult}\left(\mathcal{P}, \mathcal{E}\left(\pi_{i, j}\right)\left\lceil\varepsilon_{j}\right)\right.
$$

is illfounded shows that $\operatorname{ult}\left(\mathcal{Q}, \mathcal{E}\left(\pi_{i, j}\right)\left\lceil\varepsilon_{j}\right)\right.$ is illfounded. 田

Now let $(\overline{\mathcal{S}}, \mathcal{S})$ be a successful coiteration of $\left(\mathcal{Q}, \mathcal{P}^{*}\right)$. Both $\mathcal{Q}$ and $\mathcal{P}^{*}$ are lower-part $x$-premice, so one of $\overline{\mathcal{S}}$ and $\mathcal{S}$ is trivial, and the other is thorough. The minimality of $\mathcal{Q}$ described above, and the fact that $\operatorname{ult}\left(\mathcal{P}^{*}, \mathcal{E}\left(\pi_{i}\right)\lceil\varepsilon)\right.$ is illfounded guarantees that $\overline{\mathcal{S}}$ is trivial. Then $\mathcal{Q} \unlhd \mathcal{M}_{\infty}(\mathcal{S})$. Consider the following figure.

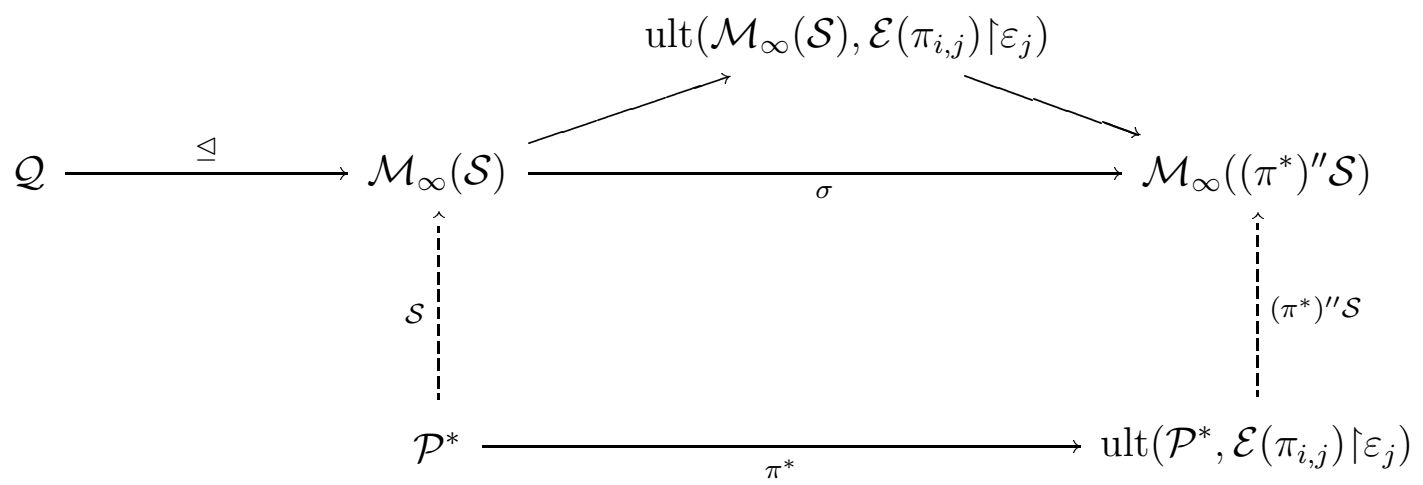

By $\pi^{*}$ we mean the ultrapower map. The vertical dashed lines represent iteration trees, but not necessarily embeddings. Recall that

\footnotetext{
${ }^{4}$ Note that thorough iteration trees on a fixed $x$-premouse are indexed by finite sets of ordinals.

${ }^{5}$ Just replace $\mathcal{P}$ by $\mathcal{Q}$ and $\mathcal{R}_{i}$ by $\operatorname{ult}\left(\mathcal{Q}, \mathcal{E}\left(\pi_{i}\right) \mid \varepsilon\right)$ two paragraphs above.
} 
$\operatorname{ult}\left(\mathcal{P}^{*}, \mathcal{E}\left(\pi_{i, j}\right)\left\lceil\varepsilon_{j}\right)\right.$ is iterable. We copy $\mathcal{S}$ using $\pi^{*}$, and let $\sigma$ be the final copying map. Then $\sigma\left\lceil\varepsilon_{i}=\pi\left\lceil\varepsilon_{i}\right.\right.$ since all critical points of extenders used on $\mathcal{S}$ are at least $\varepsilon_{i}$. So

$$
\operatorname{ult}\left(\mathcal{M}_{\infty}(\mathcal{S}), \mathcal{E}\left(\pi_{i, j}\right)\left\lceil\varepsilon_{j}\right)=\operatorname{ult}\left(\mathcal{M}_{\infty}(\mathcal{S}), \mathcal{E}(\sigma)\left\lceil\varepsilon_{j}\right)\right.\right.
$$

embeds into $\mathcal{M}_{\infty}\left(\left(\pi^{*}\right)^{\prime \prime} \mathcal{S}\right)$ as indicated. In particular,

$$
\operatorname{ult}\left(\mathcal{M}_{\infty}(\mathcal{S}), \mathcal{E}\left(\pi_{i, j}\right)\left\lceil\varepsilon_{j}\right)\right.
$$

is wellfounded.

But $\mathcal{Q} \unlhd \mathcal{M}_{\infty}(\mathcal{S})$ and $\operatorname{ult}\left(\mathcal{Q}, \mathcal{E}\left(\pi_{i, j}\right)\left\lceil\varepsilon_{j}\right)\right.$ is illfounded, so

$$
\operatorname{ult}\left(\mathcal{M}_{\infty}(\mathcal{S}), \mathcal{E}\left(\pi_{i, j}\right)\left\lceil\varepsilon_{j}\right)\right.
$$

is illfounded. This contradiction completes our consideration of Case 2, and the proof of Theorem 3.2.

\section{A WEAK ITERABILITY CONDITION}

Notice that in $\S \S 2-3$, the iterability of $W$ was used to show that all the relevant iteration trees are linear, even thorough. But, the linear iterability of $W$ does not suffice for those arguments.

Nevertheless, the assumption that $W$ is $\star$-iterable is more than is required for the proofs of Theorems 2.4 and 3.2. In this section, we sketch why internal $\star$-iterability for countable premice elementarily realizable in a level of $W$ suffices for the arguments in the preceding sections.

Definition 4.1. Let $W$ be an $x$-weasel and $\alpha$ an ordinal. We say that a structure $\mathcal{P}$ is realizable in $W$ iff $\mathcal{P}$ is an $\dot{x}^{\mathcal{P}}$-premouse and there exists an ordinal $\Omega$ and an elementary embedding of $\mathcal{P}$ into $\mathcal{J}_{\Omega}^{W}$. We say that $W$ is $\star \star$-iterable iff the following conditions hold for every countable $\mathcal{P}$ which is realizable in $W$.

(a) For every ordinal $\alpha \leq \omega_{1}, \mathcal{P}$ is $(\omega, \alpha)$-iterable in the sense of [St2, Definition 2.8].

(b) There is no infinite composition of thorough iteration trees on $\mathcal{P}$ such that each iteration tree involves a drop.

We say that $W$ is internally $\star \star$-iterable iff

$$
W \models " L[\dot{E}, \dot{x}] \text { is } \star \star \text {-iterable". }
$$

Theorem 4.2. Suppose that $W=L[\vec{E}, x]$ is an internally $\star \star$-iterable, lower-part $x$-weasel. Let $\kappa$ be a cardinal of $W$ such that

$$
(\cup x)^{+} \cup \aleph_{2} \leq \operatorname{card}(\kappa)
$$

Put $\lambda=\left(\kappa^{+}\right)^{W}$ and suppose that $\operatorname{cf}(\lambda)<\operatorname{card}(\kappa)$. Then, given

$$
\delta<\operatorname{card}(\kappa)
$$


there exists $\mu \geq \delta$ and a normal ultrafilter $U$ on $\mathcal{P}(\mu) \cap W$ such that $U$ is weakly amenable to $W$ and $\operatorname{ult}(W, U)$ is wellfounded.

Theorem 4.2 is just Theorem 3.2 restated with $\star$-iterability weakened to internal $\star \star$-iterablity; we now sketch how to modify the proof of the former to yield the latter. Let us consider the first time that iterability is used in the proof of Theorem 3.2, which is to see that in Case 1 of the proof, $\left(\overline{\mathcal{T}}_{i}, \mathcal{T}\right)$ witnesses that $\Phi\left(W_{i}, \mathcal{J}_{\Omega}^{W}\right)$ holds whenever $i \in S_{0}$. Fix $i \in S_{0}$ and suppose that $\Phi\left(W_{i}, \mathcal{J}_{\Omega}^{W}\right)$ fails. By the absoluteness of wellfoundedness, the objects listed below exist in $W$, because the objects exist in $V$ and there is a tree in $W$ that searches for them.

- a countable, transitive model $M$ of a large chunk of ZFC

- a set of ordinals $x^{M}$ in $M$, a lower-part $x^{M}$-premouse $W^{M}$ in $M$, and ordinals $\delta^{M}<\kappa^{M}<\lambda^{M}<\Omega^{M}$ in $M$, which are related as in the hypothesis of Theorem 2.4 from the point of view of $M$

- an internally approachable chain of elementary substructures of $\left(V_{\Omega^{M}+1}\right)^{M}$ of the sort that arises when we attempt to run the proof of Theorem 2.4 inside $M$

- a map $\pi^{M}: N^{M} \longrightarrow\left(V_{\Omega^{M}+1}\right)^{M}$ in $M$ which comes from collapsing one of the substructures on the internally approachable chain of $M$ to be transitive

- an $x^{M}$-premouse $\bar{W}^{M}$ such that $\pi^{M}\left(\bar{W}^{M}\right)=W^{M}$ and

$$
M \models \neg \Phi\left(\bar{W}^{M}, W^{M}\right)
$$

- an elementary embedding $\tau: W^{M} \longrightarrow \mathcal{J}_{\Omega}^{W}$

The existence of elementary embeddings $\tau \circ \pi: \bar{W}^{M} \longrightarrow \mathcal{J}_{\Omega}^{W}$ and $\tau: W^{M} \longrightarrow \mathcal{J}_{\Omega}^{W}$ in $W$ implies that $\bar{W}^{M}$ and $W^{M}$ are both countable, realizable $x^{M}$-premice in $W$, and are therefore $\star$-iterable in $W$. In particular, they are iterable in $W$, so there is a successful coiteration $(\overline{\mathcal{T}}, \mathcal{T})$ of $\left(\bar{W}^{M}, W^{M}\right)$ in $W$. Arguing as in the proof of Theorem 2.4, we see that $\overline{\mathcal{T}}$ is trivial and that $\mathcal{T}$ is thorough (in $W$, hence in $V$ ). But then $(\overline{\mathcal{T}}, \mathcal{T})$ witnesses that $M \models \Phi\left(\bar{W}^{M}, W^{M}\right)$, which is a contradiction.

There are several other applications of iterability in the proof of Theorem 3.2. All can be reduced to applications of internal $\star \star$-iterability using the preceding idea. We leave the details to the reader.

\section{The Jensen COVERING PROPERTY}

Definition 5.1. If $W$ is an $x$-weasel and $\kappa$ is an ordinal, then $C P(W, \kappa)$ holds iff $\cup x<\operatorname{card}(\kappa)$ and for every uncountable $A \subseteq \kappa$ with $\cup x \subseteq A$, there exists $B \in W$ such that $A \subseteq B$ and $\operatorname{card}(B)=\operatorname{card}(A)$. 
Definition 5.2. An $x$-weasel $W$ satisfies strong condensation iff for every $x$-premouse $\bar{W}$, if there is an elementary embedding from $\bar{W}$ into a rank initial segment of $W$, then $\bar{W} \triangleleft W$.

Theorem 5.3. Let $W=L[\vec{E}, x]$ be an internally $\star \star$-iterable, lowerpart $x$-weasel. Suppose that $\kappa$ is the least ordinal such that $C P(W, \kappa)$ fails. Let $A$ be a witness to the failure of $C P(W, \kappa)$. Then one of the following holds.

(a) There is an ordinal $\mu>\operatorname{card}(A)$ and a normal ultrafilter $U$ on $\mathcal{P}(\mu) \cap W$ such that $U$ is weakly amenable to $W$ and $\operatorname{ult}(W, U)$ is wellfounded.

(b) There exists $\mathcal{R} \triangleleft W$ with $\rho_{\omega}(\mathcal{R})=\kappa$, and there exists a set $C \subset \kappa$ of indiscernibles for $W \| \kappa$ such that $C$ is unbounded in $\kappa$ and the last extender of $\mathcal{R}$ is a measure generated by $C$. In addition,

$$
A \subset H_{\omega}^{\mathcal{R}}(\bar{\kappa} \cup C)
$$

for some $\bar{\kappa}<\kappa$.

Moreover, if $W$ satisfies strong condensation, then it is (a) that holds.

The simplest weasel that satisfies strong condensation is $L$; thus Theorem 5.3 extends Jensen's covering lemma for $L$. A more complicated example of a lower-part weasel that satisfies strong condensation is the minimal model closed under sharps for sets (assuming sharps exist). Another example is the minimal model closed under the operator which sends a set $X$ to $M_{1}(X)^{\#}$, the sharp for the iterable one Woodin cardinal model built over $X$ (assuming the operator is total). These examples of $x$-weasels are typical of those that come up in [W], in that they are minimal with respect to a certain lower-part inner model operator, and hence satisfy strong condensation.

We remark that there are examples showing the need for clause (b) in Theorem 5.3.

Proof of Theorem 5.3. Let us assume that $W$ is $\star$-iterable; the reduction to internal $\star \star$-iterability is as outlined in $\S 4$.

Let $\kappa$ be least such that $\mathrm{CP}(W, \kappa)$ fails, and let $A \subset \kappa$ witness the failure of $\operatorname{CP}(W, \kappa)$. Let $\varepsilon=(\operatorname{card}(A))^{+}$. Then

$$
\operatorname{cf}(\kappa) \leq \operatorname{card}(A)<\varepsilon \leq \operatorname{card}(\kappa) \leq \kappa=\sup (A) .
$$

Since $\kappa$ is least such that $\operatorname{CP}(W, \kappa)$ fails, $\kappa$ is a cardinal in $W$.

In this proof, $\kappa$ will play the role that $\lambda$ played in the proofs of our other theorems. Note that by Theorem 3.2, we may assume that $\kappa$ is a limit cardinal of $W$. 
Let $\Omega>\kappa$ be a regular cardinal. Let $\left\langle X_{i} \mid i<\varepsilon\right\rangle$ be a continuous chain of elementary substructures of $V_{\Omega+1}$ such that for all $j<\varepsilon$,

$$
\begin{gathered}
\operatorname{card}\left(X_{j}\right)=\operatorname{card}(A), \\
\left\langle X_{i} \mid i \leq j\right\rangle \in X_{j+1},
\end{gathered}
$$

and

$$
X_{j} \cap \varepsilon \in \varepsilon \text {. }
$$

For $i<\varepsilon$, let $\varepsilon_{i}=X_{i} \cap \varepsilon_{i}$ and $\pi_{i}: N_{i} \longrightarrow V_{\Omega+1}$ be the uncollapse of $X_{i}$, so that $\operatorname{crit}\left(\pi_{i}\right)=\varepsilon_{i}$. We also have or assume that

$$
(\cup x) \cup \operatorname{card}(A) \cup\left\{\kappa, \mathcal{J}_{\Omega}^{W}, A\right\} \in X_{0} .
$$

Say $\pi_{i}\left(\kappa_{i}\right)=\kappa$ and $\pi_{i}\left(W_{i}\right)=\mathcal{J}_{\Omega}^{W}$ whenever $i<\varepsilon$.

Case 1. There is a stationary set

$$
S_{0} \subseteq\left\{i<\varepsilon \mid \operatorname{cf}(i)>\omega \wedge i=\varepsilon_{i}\right\}
$$

such that $i \in S_{0}$ implies $\left(\varepsilon_{i}^{+}\right)^{W_{i}}<\left(\varepsilon_{i}^{+}\right)^{W}$.

For $i \in S_{0}$, let $\left(\overline{\mathcal{T}}_{i}, \mathcal{T}_{i}\right)$ be a successful coiteration of $\left(W_{i}, \mathcal{J}_{\Omega}^{W}\right)$, and say this coiteration has length $\theta_{i}$. Then $\overline{\mathcal{T}}_{i}$ is trivial and $\mathcal{T}_{i}$ is thorough whenever $i \in S_{0}$. In particular, $\Phi\left(W_{i}, \mathcal{J}_{\Omega}^{W}\right)$ holds.

Notice that if $W$ satisfies strong condensation, then $\bar{W} \triangleleft W$, so $\mathcal{T}_{i}$ is also trivial.

For $i \in S_{0}$, let $\eta_{i}$ be the least ordinal $\eta$ such that $\operatorname{crit}\left(E_{\eta}^{\mathcal{T}}\right) \geq \kappa_{i}$, if such an ordinal exists, and let $\eta_{i}$ be $\theta_{i}$ otherwise. For $i \in S_{0}$, let

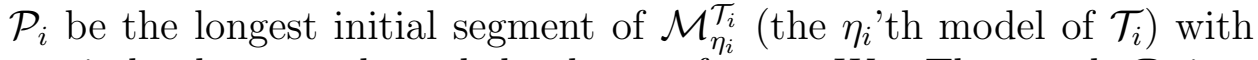
precisely the same bounded subsets of $\kappa_{i}$ as $W_{i}$. Then each $\mathcal{P}_{i}$ is a $\kappa_{i}$-sound $x$-premouse.

For $i \in S_{0}$, let $\mathcal{R}_{i}=\operatorname{ult}\left(\mathcal{P}_{i}, \mathcal{E}\left(\pi_{i}\right)\lceil\kappa)\right.$. Recall that this ultrapower is defined using coordinates $a \in[\kappa]^{<\omega}$, and consists of equivalence classes of certain $\mathcal{P}_{i}$-definable functions of the form $f:[\alpha]^{|a|} \longrightarrow\left|\mathcal{P}_{i}\right|$ with $\alpha<\kappa_{i}$. This makes sense because $\mathcal{P}_{i}$ and $W_{i}$ have the same bounded subsets of $\kappa_{i}$, and because $\pi_{i}$ is continuous at $\kappa_{i}$.

Claim 5.3.1. There is a $i \in S_{0}$ such that either $\Phi\left(\mathcal{J}_{\Omega}^{W}, \mathcal{R}_{i}\right)$ holds or $\mathcal{R}_{i} \triangleleft W$.

Before proving Claim 5.3.1, let us see how to use it to finish the proof of Theorem 5.3 under Case 1. Fix $i$ as in Claim 5.3.1. To ease the notation, let us put $\pi=\pi_{i}, \bar{W}=W_{i}, \bar{\kappa}=\kappa_{i}, \eta=\eta_{i}, \mathcal{T}=\mathcal{T}_{i}$, $\mathcal{P}=\mathcal{P}_{i}$, and $\mathcal{R}=\mathcal{R}_{i}$ until we start the proof of Claim 5.3.1.

If $\Phi(W, \mathcal{R})$ holds, then (a) holds by an argument as in the proof of Theorem 2.4. We can also conclude that (a) holds if for a proper 
class of $\Omega$, there is a corresponding $i(\Omega)$ such that $\Phi\left(\mathcal{J}_{\Omega}^{W}, \mathcal{R}_{i(\Omega)}\right)$ holds. This is by a counting argument, using that there are only set many possibilities for $\mathcal{R}_{i(\Omega)}$.

So assume that $\mathcal{R} \triangleleft W$. In particular, $\mathcal{R} \triangleleft \mathcal{J}_{\Omega}^{W}$ and $\mathcal{R}$ is an element of $W$.

We claim that $\mathcal{P}=\mathcal{M}_{\eta}^{\mathcal{T}}$. Suppose not. Then $\mathcal{P}$ is a fully sound proper initial segment of $\mathcal{M}_{\eta}^{\mathcal{T}}$, and

$$
A \subseteq \operatorname{ran}(\pi) \cap \kappa \subset H_{n+1}^{\mathcal{R}}\left(\rho_{n+1}(\mathcal{P}) \cup \pi\left(p_{n+1}(\mathcal{P})\right)\right) \subseteq H_{\omega}^{\mathcal{R}}(\bar{\kappa})
$$

where $n=n(\mathcal{P}, \bar{\kappa})$. But $H_{\omega}^{\mathcal{R}}(\bar{\kappa})$ is an element of $W$ and has the same cardinality as $A$. This contradicts the assumption that $A$ witnesses the failure of $\operatorname{CP}(W, \kappa)$. Therefore $\mathcal{P}=\mathcal{M}_{\eta}^{\mathcal{T}}$.

In particular, $\eta \neq 0$, so we are done with Case 1 if $W$ satisfies strong condensation.

Let

$$
\bar{C}=\left\{\operatorname{crit}\left(E_{\gamma}^{\mathcal{T}}\right) \mid 0 \leq \gamma<\eta \wedge \eta \cap \mathcal{D}^{\mathcal{T}} \subseteq \gamma\right\}
$$

Then

$$
\mathcal{P}=\mathcal{H}_{n+1}^{\mathcal{P}}\left(\rho_{n+1}(\mathcal{P}) \cup \bar{C} \cup p_{n+1}(\mathcal{P})\right)
$$

where $n=n(\mathcal{P}, \bar{\kappa})$. So

$$
\begin{aligned}
A \subseteq \operatorname{ran}(\pi) \cap \kappa \subset H_{n+1}^{\mathcal{R}}\left(\rho_{n+1}(\mathcal{P}) \cup\left(\pi^{\prime \prime} \bar{C}\right) \cup \pi\left(p_{n+1}(\mathcal{P})\right)\right) \\
\subseteq H_{\omega}^{\mathcal{R}}\left(\bar{\kappa} \cup\left(\pi^{\prime \prime} \bar{C}\right)\right)
\end{aligned}
$$

Suppose for contradiction that $\bar{C}$ is bounded in $\bar{\kappa}$. Equivalently, $\pi^{\prime \prime} \bar{C}$ is bounded in $\kappa$. Since $\kappa$ is least such that $\operatorname{CP}(W, \kappa)$ fails,

$$
\mathrm{CP}\left(W, \sup \left(\pi^{\prime \prime} \bar{C}\right)\right)
$$

holds. So there is a $B \in W$ such that $\omega_{1} \cup(\cup x) \cup\left(\pi^{\prime \prime} \bar{C}\right) \subseteq B$ and

$$
\operatorname{card}(B) \leq \operatorname{card}\left(\omega_{1} \cup(\cup x) \cup\left(\pi^{\prime \prime} \bar{C}\right)\right) \leq \operatorname{card}(A) .
$$

But then $H_{\omega}^{\mathcal{R}}(\bar{\kappa} \cup B)$ is a set in $W$ containing $A$, and $H_{\omega}^{\mathcal{R}}(\bar{\kappa} \cup B)$ has the same cardinality as $A$. This contradicts that $A$ is a counterexample to $\mathrm{CP}(W, \kappa)$. Therefore $\bar{\kappa}=\sup (\bar{C})$.

It follows from the fact that $\mathcal{T}$ is thorough that $\eta$ is a limit ordinal, $\bar{C}$ is a set of indiscernibles for $\mathcal{J}_{\bar{\kappa}}^{\mathcal{P}}=\bar{W} \| \bar{\kappa}$, and the last extender of $\mathcal{P}$ is a measure generated by $\bar{C}$. By the elementarity of the ultrapower map from $\mathcal{P}$ into $\mathcal{R}$ and its agreement with $\pi \mid \bar{\kappa}$, we conclude that clause (b) of Theorem 5.3 holds with $C=\pi^{\prime \prime} \bar{C}$.

Proof of Claim 5.3.1. Assume for contradiction that Claim 5.3.1 is false. So for every $i \in S_{0}$, either $\Phi\left(W, \mathcal{R}_{i}\right)$ fails or $\mathcal{R}_{i} \nless W$.

Let $S$ be a stationary subset of $S_{0}$ on which the choice functions $i \mapsto \eta_{i}$ and $i \mapsto n\left(\mathcal{P}_{i}, \kappa_{i}\right)$ are constant. Such a set $S$ exists by Fodor's 
lemma MiSch, Lemma 1.1]. Say $\eta=\eta_{i}$ and $n\left(\mathcal{P}_{i}, \kappa_{i}\right)=n$ whenever $i \in S$.

Let $j \in \lim (S) \cap S$. Let $\psi: M \longrightarrow V_{\Omega+2}$ be an elementary embedding with $M$ countable and transitive, and everything relevant in the range of $\psi$. Since $\psi$ is countable, we may fix $i \in j \cap S$ such that

$$
\operatorname{ran}(\psi) \cap X_{j} \subset X_{i} .
$$

Let $\pi_{i, j}: N_{i} \longrightarrow N_{j}$ be the uncollapse of $\left(\pi_{j}\right)^{-1}\left(X_{i}\right)$.

We claim that $\operatorname{ran}(\psi) \cap \kappa$ is unbounded in $\kappa$. The proof is essentially the same as that of Claim 3.1.1, and we leave the details to the reader.

Therefore

$$
\sup \left(\left(\pi_{i, j}\right)^{\prime \prime} \kappa_{i}\right)=\pi_{i, j}\left(\kappa_{i}\right)=\kappa_{j}
$$

Arguing as in MiSch, Lemma 2.1], we find a "pull-back" $\mathcal{P}_{j}^{*}$ of $\mathcal{P}_{j}$ by $\pi_{i, j}$. That is, we find an $x$-premouse $\mathcal{P}_{j}^{*}$ such that $\mathcal{P}_{j}^{*}$ agrees with $W_{i}$ below $\kappa_{i}$ and

$$
\mathcal{P}_{j}=\operatorname{ult}\left(\mathcal{P}_{j}^{*}, \mathcal{E}\left(\pi_{i, j}\right)\left\lceil\kappa_{j}\right) .\right.
$$

Moreover, $\mathcal{P}_{j}^{*}$ is $\kappa_{i}$-sound with $n\left(\mathcal{P}_{j}^{*}, \kappa_{i}\right)=n$.

Since both $\mathcal{P}_{i}$ and $\mathcal{P}_{j}^{*}$ are $\kappa_{i}$-sound and iterable above $\kappa_{i}$, one must be an initial segment of the other. Let $\mathcal{P}$ be the shorter of $\mathcal{P}_{i}$ and $\mathcal{P}_{j}^{*}$. It will be important that $\mathcal{P} \in X_{j}$ and that $\mathcal{P} \unlhd \mathcal{P}_{j}^{*}$.

Now $\operatorname{ult}\left(\mathcal{P}, \mathcal{E}\left(\pi_{i}\right)\lceil\kappa)\right.$ is "bad" in the sense that

$$
\Phi\left(\mathcal{J}_{\Omega}^{W}, \operatorname{ult}\left(\mathcal{P}, \mathcal{E}\left(\pi_{i}\right)\lceil\kappa)\right)\right.
$$

fails and $\operatorname{ult}\left(\mathcal{P}, \mathcal{E}\left(\pi_{i}\right)\lceil\kappa) \pitchfork \mathcal{J}_{\Omega}^{W}\right.$.

Therefore,

$$
N_{j} \models \neg \Phi\left(\left(\pi_{j}\right)^{-1}\left(\mathcal{J}_{\Omega}^{W}\right),\left(\pi_{j}\right)^{-1}\left(\operatorname{ult}\left(\mathcal{P}, \mathcal{E}\left(\pi_{i}\right)\lceil\kappa)\right)\right)\right.
$$

and

$$
N_{j} \models\left(\pi_{j}\right)^{-1}\left(\operatorname{ult}\left(\mathcal{P}, \mathcal{E}\left(\pi_{i}\right)\lceil\kappa)\right) \Varangle\left(\pi_{j}\right)^{-1}\left(\mathcal{J}_{\Omega}^{W}\right)\right.
$$

Hence,

$$
N_{j} \models \neg \Phi\left(W_{j}, \operatorname{ult}\left(\mathcal{P}, \mathcal{E}\left(\pi_{i, j}\left\lceil\kappa_{j}\right)\right)\right)\right.
$$

and

$$
N_{j} \models \operatorname{ult}\left(\mathcal{P}, \mathcal{E}\left(\pi_{i, j}\left\lceil\kappa_{j}\right)\right) \nless W_{j} .\right.
$$

By absoluteness, $\Phi\left(W_{j}\right.$, ult $\left(\mathcal{P}, \mathcal{E}\left(\pi_{i, j}\left\lceil\kappa_{j}\right)\right)\right)$ fails and

$$
\operatorname{ult}\left(\mathcal{P}, \mathcal{E}\left(\pi_{i, j}\left\lceil\kappa_{j}\right)\right) 丸 W_{j} .\right.
$$

Now $\operatorname{ult}\left(\mathcal{P}, \mathcal{E}\left(\pi_{i, j}\left\lceil\kappa_{j}\right)\right)\right.$ is iterable above $\kappa_{j}$ since there is a sufficiently elementary embedding from $\operatorname{ult}\left(\mathcal{P}, \mathcal{E}\left(\pi_{i, j}\left\lceil\kappa_{j}\right)\right)\right.$ into an initial segment of

$$
\mathcal{P}_{j}=\operatorname{ult}\left(\mathcal{P}_{j}^{*}, \mathcal{E}\left(\pi_{i, j}\left\lceil\kappa_{j}\right)\right),\right.
$$

and the critical point of the embedding is at least $\kappa_{j}$. 
Moreover, $\operatorname{ult}\left(\mathcal{P}, \mathcal{E}\left(\pi_{i, j}\left\lceil\kappa_{j}\right)\right)\right.$ is $\kappa_{j}$-sound and agrees with $W_{j}$ below $\kappa_{j}$. In the standard way, from the fact that we can compare

$$
\operatorname{ult}\left(\mathcal{P}, \mathcal{E}\left(\pi_{i, j}\left\lceil\kappa_{j}\right)\right)\right.
$$

and $W_{j}$, we conclude that either $\Phi\left(W_{j}, \operatorname{ult}\left(\mathcal{P}, \mathcal{E}\left(\pi_{i, j}\left\lceil\kappa_{j}\right)\right)\right)\right.$ holds or $\operatorname{ult}\left(\mathcal{P}, \mathcal{E}\left(\pi_{i, j}\left\lceil\kappa_{j}\right)\right) \triangleleft W_{j}\right.$. This is a contradiction.

Case 2. There is a stationary set

$$
S_{0} \subseteq\left\{i<\varepsilon \mid \operatorname{cf}(i)>\omega \wedge i=\varepsilon_{i}\right\}
$$

such that $i \in S_{0}$ implies $\left(\varepsilon_{i}^{+}\right)^{W_{i}}=\left(\varepsilon_{i}^{+}\right)^{W}$.

In this case, we conclude that (a) holds by an argument just like that given in Case 2 of the proof of Theorem 3.2.

\section{REFERENCES}

[De] K.J. Devlin, Constructibility, Springer-Verlag (1984).

[Do] A.J. Dodd, The core model, London Mathematical Society Lecture Notes Series 61, Cambridge University Press (1982).

[DoJe1] A.J. Dodd and R.B. Jensen, The core model, Annals of Mathematical Logic 20 (1981) 43-75.

[DoJe2] A.J. Dodd and R.B. Jensen, The covering lemma for K, Annals of Mathematical Logic 22 (1982) 1-30.

[DoJe3] A.J. Dodd and R.B. Jensen, The covering lemma for $L[U]$, Annals of Mathematical Logic 22 (1982) 127-135.

[Je] R.B. Jensen, The fine structure of the constructible hierarchy, Annals of Mathematical Logic 4 (1972) 229-308.

$[\mathrm{KW}] \quad$ A. Kechris and W.H. Woodin, Equivalence of partition properties and determinacy, Proceedings of the National Academy of Science USA 80 (1983).

[MaSt] D.A. Martin and J.R. Steel, Iteration trees, Journal of the American Mathematical Society 7 (1994) 1-73.

[MiSch] W.J. Mitchell and E. Schimmerling, Covering without countable closure, Mathematical Research Letters 2 (1995) 595-609.

[MiSchSt] W.J. Mitchell, E. Schimmerling, and J.R. Steel, The covering lemma up to a Woodin cardinal, to appear in Annals of Pure and Applied Logic.

[MiSt] W.J. Mitchell and J.R. Steel, Fine structure and iteration trees, Lecture Notes in Logic 3, Springer-Verlag (1994).

[Sch1] E. Schimmerling, Combinatorial principles in the core model for one Woodin cardinal, Annals of Pure and Applied Logic 74 (1995) 153-201.

[Sch2] E. Schimmerling, $\square_{\kappa}^{<\omega}$ holds in $L[\vec{E}]$, submitted to The Journal of Symbolic Logic.

[SchSt1] E. Schimmerling and J.R. Steel, Fine structure for tame inner models, The Journal of Symbolic Logic 61 (1996) 621-639.

[SchSt2] E. Schimmerling and J.R. Steel, The maximality of the core model, preprint. 
[St1] J.R. Steel, Scales in $L(\mathbb{R})$, in Cabal Seminar 79-81, Lecture Notes in Mathematics 1019 (1983).

[St2] J.R. Steel, The core model iterability problem, to appear in Lecture Notes in Logic, Springer-Verlag.

[St3] J.R. Steel, Inner models with many Woodin cardinals, Annals of Pure Applied Logic 65 (1993) 185-209.

[St4] J.R. Steel, Core models with more Woodin cardinals, preprint.

[W] W.H. Woodin, lectures at the 1995 Greater Boston Logic Conference, workshop on Inner Model Theory.

Department of Mathematics, University of California, Irvine, CA 92697-4065, USA

E-mail address: eschimme@math.uci.edu

Department of Mathematics, University of California, Berkeley, CALIFORNIA 94720, USA

E-mail address: woodin@math.berkeley.edu 